\title{
Supplementary Material: A Comparative Ab-Initio Study of Substituted Norbornadiene-Quadricyclane Compounds for Solar Thermal Storage
}

\author{
Mikael J. Kuisma, ${ }^{* \dagger}$ Angelica M. Lundin,${ }^{\ddagger}$ Kasper Moth-Poulsen, ${ }^{\ddagger}$ Per \\ Hyldgaard, and Paul Erhart*,† \\ Department of Physics, Chalmers University of Technology, Gothenburg, Sweden, \\ Department of Chemistry and Chemical Engineering, Chalmers University of Technology, \\ Gothenburg, Sweden, and Department of Microtechnology and Nanoscience, Chalmers \\ University of Technology, Gothenburg, Sweden \\ E-mail: mikael.kuisma@chalmers.se; erhart@chalmers.se
}

\section{Molecular structure of the un- substituted compound (1)}

Detailed comparison unsubstituted norbornadiene and quadricyclane is presented in Tables 1 and 2 .

\section{Compilation of results from calculations of storage energy}

The storage energies (electronic energy difference between $\mathrm{N}$ and $\mathrm{Q}$ ) computed using CASPT2 as well as DFT are compiled in Table 3 .

${ }^{*}$ To whom correspondence should be addressed

${ }^{\dagger}$ Department of Physics, Chalmers University of Technology, Gothenburg, Sweden

${ }^{\ddagger}$ Department of Chemistry and Chemical Engineering, Chalmers University of Technology, Gothenburg, Sweden

IDepartment of Microtechnology and Nanoscience, Chalmers University of Technology, Gothenburg, Sweden

\section{Compilation of results from calculations of absorption be- havior}

The first excitation energies obtained from CASPT2 calculations are compiled in Table 4. Excitation energies and dipole strengths from TD-DFT calculations for the first five excitations of the molecules considered in this study are compiled in Tables 5 and 6 . Figure 1 shows the molar attenuation coefficients in toluene obtained using different exchange-correlation functionals, which are based on the data presented in Table 6 .

\section{Convergence of MP2 and $\operatorname{CCSD}(\mathrm{T})$ calculations with basis set}

As a further benchmark of our calculations, we performed an accurate comparison of computational and experimental results regarding the geometry and the gas-phase thermochemistry of the unsubstituted norbornadiene quadricyclane system (1). All geometries were fully relaxed 
Table 1: Bond lengths $r$ in $\AA$ and angles $\theta$ of norbornadiene compared to experimental values.

\begin{tabular}{lcccc}
\hline \hline $\mathrm{N}$ & $r(\mathrm{C} 2-\mathrm{C} 3)$ & $r(\mathrm{C} 1-\mathrm{C} 2)$ & $r(\mathrm{C} 1-\mathrm{C} 7)$ & $\theta(\mathrm{C} 1, \mathrm{C} 4, \mathrm{C} 7)$ \\
\hline Experiment 1 & 1.3362 & 1.5304 & 1.5567 & \\
Experiment 2 ${ }^{a}$ & 1.338 & 1.533 & 1.571 & $92.2^{\circ}$ \\
$\mathrm{PBE}$ & 1.343 & 1.544 & 1.562 & $92.2^{\circ}$ \\
B3LYP & 1.334 & 1.544 & 1.561 & $92.0^{\circ}$ \\
B3LYP (aug-cc-vtzp) & 1.329 & 1.540 & 1.558 & $92.0^{\circ}$ \\
MP2 (aug-cc-vdzp) & 1.358 & 1.542 & 1.564 & $92.4^{\circ}$ \\
MP2 (aug-cc-vtzp) & 1.342 & 1.532 & 1.553 & $92.3^{\circ}$ \\
M06 & 1.329 & 1.532 & 1.546 & $92.2^{\circ}$ \\
\hline
\end{tabular}

${ }^{a}$ Data from fit I; fit II yields and angle $\theta(\mathrm{C} 1, \mathrm{C} 4, \mathrm{C} 7)$ of $96^{\circ}$, which is not supported by the computational data.

Table 2: Bond lengths $r$ in $\AA$ and angles $\theta$ of quadricyclane compared to experimental values.

\begin{tabular}{|c|c|c|c|}
\hline $\mathrm{Q}$ & $r(\mathrm{C}-\mathrm{C}$ (short) $)$ & $r(\mathrm{C}-\mathrm{C}($ long $))$ & $\theta(\mathrm{C} 1, \mathrm{C} 7, \mathrm{C} 4)$ \\
\hline Experiment $^{3}$ & 1.518 & 1.562 & $98.50^{\circ}$ \\
\hline PBE & 1.522 & 1.551 & $98.88^{\circ}$ \\
\hline B3LYP & 1.517 & 1.550 & $98.85^{\circ}$ \\
\hline B3LYP (aug-cc-vtzp) & 1.513 & 1.547 & $98.82^{\circ}$ \\
\hline MP2 (aug-cc-vdzp) & 1.533 & 1.552 & $98.92^{\circ}$ \\
\hline MP2 (aug-cc-vtzp) & 1.519 & 1.540 & $98.84^{\circ}$ \\
\hline M06 & 1.507 & 1.538 & $98.85^{\circ}$ \\
\hline
\end{tabular}

Table 3: Storage energies in $\mathrm{kJ} / \mathrm{mol}$ from CASPT2 as well as DFT calculations.

\begin{tabular}{lcrrrrr}
\hline \hline Compound & $\mathbf{1}$ & $\mathbf{2}$ & $\mathbf{3}$ & $\mathbf{4}$ & $\mathbf{5}$ & $\mathbf{6}$ \\
\hline & \multicolumn{2}{l}{ Vacuum } & & & & \\
\hline CASPT2//CASSCF & 91 & 100 & 101 & 99 & 99 & 99 \\
CASPT2//PBE & 94 & 96 & 97 & 98 & 97 & 102 \\
PBE & 71 & 91 & 87 & 86 & 86 & 90 \\
B3LYP & 99 & 115 & 113 & 112 & 112 & 115 \\
CAM-B3LYP & 87 & 100 & 98 & 96 & 96 & 99 \\
M06-L & 41 & 71 & 61 & 69 & 67 & 67 \\
M06 & 53 & 62 & 68 & 68 & 67 & 74 \\
M06-2x & 65 & 75 & 76 & 73 & 75 & 74 \\
\hline & Toluene & & & & \\
\hline PBE & 64 & 86 & 83 & 82 & 83 & 87 \\
B3LYP & 93 & 111 & 109 & 108 & 108 & 112 \\
CAM-B3LYP & 80 & 96 & 94 & 93 & 93 & 96 \\
\hline \hline
\end{tabular}


Table 4: Excitation energies under vacuum conditions in eV from CASPT2 calculations.

\begin{tabular}{lcccccc}
\hline \hline Compound & $\mathbf{1}$ & $\mathbf{2}$ & $\mathbf{3}$ & $\mathbf{4}$ & $\mathbf{5}$ & $\mathbf{6}$ \\
\hline CASPT2//CASSCF & 5.32 & 4.47 & 4.40 & 4.29 & 4.22 & 3.96 \\
CASPT2//PBE & 5.32 & 4.15 & 4.04 & 3.94 & 3.82 & 3.66 \\
\hline \hline
\end{tabular}

Table 5: Excitation energies in $\mathrm{eV}$ and dipole strengths (given in brackets) in vacuum from TD-DFT calculations using different exchange-correlation functionals.

\begin{tabular}{|c|c|c|c|c|c|c|c|c|c|c|c|}
\hline \multicolumn{12}{|c|}{ PBE } \\
\hline 1 & $\mathrm{~N}$ & 4.47 & $(0.00)$ & 5.00 & $(0.01)$ & 5.19 & $(0.01)$ & 5.76 & $(0.00)$ & 5.86 & $(0.00)$ \\
\hline 1 & $\mathrm{Q}$ & 5.00 & $(0.00)$ & 5.44 & $(0.00)$ & 5.51 & $(0.00)$ & 5.62 & $(0.01)$ & 6.06 & $(0.02)$ \\
\hline 2 & $\mathrm{~N}$ & 3.21 & $(0.17)$ & 3.71 & $(0.02)$ & 3.80 & $(0.06)$ & 4.01 & $(0.01)$ & 4.03 & $(0.02)$ \\
\hline 2 & $\mathrm{Q}$ & 4.10 & $(0.00)$ & 4.11 & $(0.00)$ & 4.25 & $(0.00)$ & 4.38 & $(0.00)$ & 4.57 & $(0.01)$ \\
\hline 3 & $\mathrm{~N}$ & 3.05 & $(0.18)$ & 3.54 & $(0.04)$ & 3.79 & $(0.00)$ & 3.85 & $(0.01)$ & 3.92 & $(0.04)$ \\
\hline 3 & $\mathrm{Q}$ & 3.73 & $(0.01)$ & 3.85 & $(0.05)$ & 3.98 & $(0.01)$ & 4.22 & $(0.05)$ & 4.44 & $(0.04)$ \\
\hline 4 & $\mathrm{~N}$ & 2.86 & $(0.18)$ & 3.46 & $(0.02)$ & 3.62 & $(0.01)$ & 3.84 & $(0.06)$ & 3.85 & $(0.00)$ \\
\hline 4 & $\mathrm{Q}$ & 3.40 & $(0.04)$ & 3.65 & $(0.01)$ & 3.97 & $(0.03)$ & 4.05 & $(0.04)$ & 4.24 & $(0.01)$ \\
\hline 5 & $\mathrm{~N}$ & 2.67 & $(0.21)$ & 3.42 & $(0.01)$ & 3.43 & $(0.02)$ & 3.68 & $(0.14)$ & 3.80 & $(0.01)$ \\
\hline 5 & Q & 3.06 & $(0.05)$ & 3.60 & $(0.00)$ & 3.70 & $(0.10)$ & 4.00 & $(0.01)$ & 4.18 & $(0.01)$ \\
\hline 6 & $\mathrm{~N}$ & 2.54 & $(0.23)$ & 3.16 & $(0.02)$ & 3.41 & $(0.03)$ & 3.58 & $(0.01)$ & 3.68 & $(0.01)$ \\
\hline 6 & $\mathrm{Q}$ & 2.78 & $(0.02)$ & 3.16 & $(0.01)$ & 3.78 & $(0.12)$ & 3.85 & $(0.02)$ & 3.96 & $(0.01)$ \\
\hline \multicolumn{12}{|c|}{ B3LYP } \\
\hline 1 & $\mathrm{~N}$ & 4.86 & $(0.00)$ & 5.56 & $(0.01)$ & 5.58 & $(0.01)$ & 6.15 & $(0.00)$ & 6.15 & $(0.02)$ \\
\hline 1 & $\mathrm{Q}$ & 5.47 & $(0.00)$ & 5.84 & $(0.00)$ & 5.95 & $(0.00)$ & 6.08 & $(0.01)$ & 6.53 & $(0.02)$ \\
\hline 2 & $\mathrm{~N}$ & 3.70 & $(0.22)$ & 4.32 & $(0.02)$ & 4.53 & $(0.05)$ & 4.63 & $(0.02)$ & 4.70 & $(0.03)$ \\
\hline 2 & Q & 4.94 & $(0.00)$ & 5.06 & $(0.00)$ & 5.12 & $(0.00)$ & 5.13 & $(0.00)$ & 5.23 & $(0.00)$ \\
\hline 3 & $\mathrm{~N}$ & 3.58 & $(0.22)$ & 4.17 & $(0.04)$ & 4.49 & $(0.01)$ & 4.60 & $(0.07)$ & 4.65 & $(0.02)$ \\
\hline 3 & $\mathrm{Q}$ & 4.54 & $(0.03)$ & 4.67 & $(0.06)$ & 4.83 & $(0.04)$ & 5.01 & $(0.05)$ & 5.11 & $(0.00)$ \\
\hline 4 & $\mathrm{~N}$ & 3.40 & $(0.23)$ & 4.11 & $(0.03)$ & 4.45 & $(0.03)$ & 4.49 & $(0.01)$ & 4.54 & $(0.07)$ \\
\hline 4 & Q & 4.28 & $(0.08)$ & 4.53 & $(0.01)$ & 4.75 & $(0.02)$ & 4.81 & $(0.07)$ & 5.00 & $(0.02)$ \\
\hline 5 & $\mathrm{~N}$ & 3.23 & $(0.26)$ & 4.07 & $(0.01)$ & 4.29 & $(0.03)$ & 4.36 & $(0.14)$ & 4.46 & $(0.01)$ \\
\hline 5 & Q & 3.99 & $(0.11)$ & 4.47 & $(0.08)$ & 4.51 & $(0.07)$ & 4.74 & $(0.02)$ & 4.90 & $(0.02)$ \\
\hline 6 & $\mathrm{~N}$ & 3.11 & $(0.27)$ & 3.88 & $(0.04)$ & 4.16 & $(0.05)$ & 4.26 & $(0.01)$ & 4.35 & $(0.02)$ \\
\hline 6 & Q & 3.72 & $(0.03)$ & 4.10 & $(0.02)$ & 4.36 & $(0.03)$ & 4.43 & $(0.06)$ & 4.49 & $(0.11)$ \\
\hline \multicolumn{12}{|c|}{ CAM-B3LYP } \\
\hline 1 & $\mathrm{~N}$ & 5.21 & $(0.00)$ & 6.08 & $(0.02)$ & 6.12 & $(0.03)$ & 6.54 & $(0.03)$ & 6.71 & $(0.00)$ \\
\hline 1 & Q & 5.97 & $(0.00)$ & 6.30 & $(0.00)$ & 6.48 & $(0.00)$ & 6.62 & $(0.00)$ & 7.15 & $(0.02)$ \\
\hline 2 & $\mathrm{~N}$ & 4.17 & $(0.24)$ & 4.90 & $(0.02)$ & 5.15 & $(0.00)$ & 5.29 & $(0.09)$ & 5.43 & $(0.11)$ \\
\hline 2 & Q & 5.38 & $(0.00)$ & 5.41 & $(0.00)$ & 5.85 & $(0.09)$ & 5.87 & $(0.03)$ & 6.05 & $(0.05)$ \\
\hline 3 & $\mathrm{~N}$ & 4.10 & $(0.25)$ & 4.74 & $(0.05)$ & 5.04 & $(0.01)$ & 5.32 & $(0.12)$ & 5.39 & $(0.16)$ \\
\hline 3 & $\mathrm{Q}$ & 5.00 & $(0.02)$ & 5.25 & $(0.00)$ & 5.38 & $(0.18)$ & 5.68 & $(0.20)$ & 5.73 & $(0.03)$ \\
\hline 4 & $\mathrm{~N}$ & 3.97 & $(0.27)$ & 4.74 & $(0.04)$ & 5.01 & $(0.01)$ & 5.26 & $(0.16)$ & 5.38 & $(0.17)$ \\
\hline 4 & $\mathrm{Q}$ & 5.00 & $(0.02)$ & 5.19 & $(0.10)$ & 5.20 & $(0.11)$ & 5.60 & $(0.26)$ & 5.80 & $(0.02)$ \\
\hline 5 & $\mathrm{~N}$ & 3.84 & $(0.32)$ & 4.70 & $(0.03)$ & 4.96 & $(0.02)$ & 5.11 & $(0.28)$ & 5.30 & $(0.01)$ \\
\hline 5 & $\mathrm{Q}$ & 4.88 & $(0.27)$ & 5.03 & $(0.07)$ & 5.07 & $(0.03)$ & 5.53 & $(0.23)$ & 5.62 & $(0.09)$ \\
\hline 6 & $\mathrm{~N}$ & 3.76 & $(0.35)$ & 4.46 & $(0.06)$ & 4.85 & $(0.03)$ & 4.89 & $(0.01)$ & 5.05 & $(0.21)$ \\
\hline 6 & $\mathrm{Q}$ & 4.61 & $(0.03)$ & 4.89 & $(0.23)$ & 4.92 & $(0.01)$ & 5.20 & $(0.02)$ & 5.30 & $(0.34)$ \\
\hline
\end{tabular}


Table 6: Excitation energies in $\mathrm{eV}$ and dipole strengths (given in brackets) in toluene from TD-DFT calculations using different exchange-correlation functionals.

\begin{tabular}{|c|c|c|c|c|c|c|c|c|c|c|c|}
\hline \multicolumn{12}{|c|}{$\mathrm{PBE}$} \\
\hline 1 & $\mathrm{~N}$ & 4.48 & $(0.00)$ & 5.01 & $(0.01)$ & 5.33 & $(0.01)$ & 5.93 & $(0.00)$ & 5.99 & $(0.00)$ \\
\hline 1 & Q & 5.11 & $(0.00)$ & 5.49 & $(0.00)$ & 5.63 & $(0.00)$ & 5.70 & $(0.01)$ & 6.17 & $(0.02)$ \\
\hline 2 & $\mathrm{~N}$ & 3.22 & $(0.17)$ & 3.70 & $(0.02)$ & 3.80 & $(0.07)$ & 4.00 & $(0.01)$ & 4.04 & $(0.02)$ \\
\hline 2 & $\mathrm{Q}$ & 4.00 & $(0.00)$ & 4.00 & $(0.01)$ & 4.14 & $(0.00)$ & 4.27 & $(0.00)$ & 4.54 & $(0.01)$ \\
\hline 3 & $\mathrm{~N}$ & 3.05 & $(0.18)$ & 3.54 & $(0.04)$ & 3.79 & $(0.00)$ & 3.85 & $(0.01)$ & 3.91 & $(0.05)$ \\
\hline 3 & $\mathrm{Q}$ & 3.67 & $(0.01)$ & 3.79 & $(0.06)$ & 3.92 & $(0.00)$ & 4.18 & $(0.05)$ & 4.39 & $(0.03)$ \\
\hline 4 & $\mathrm{~N}$ & 2.83 & $(0.19)$ & 3.44 & $(0.02)$ & 3.57 & $(0.01)$ & 3.83 & $(0.09)$ & 3.84 & $(0.00)$ \\
\hline 4 & $\mathrm{Q}$ & 3.29 & $(0.05)$ & 3.57 & $(0.01)$ & 3.90 & $(0.04)$ & 3.97 & $(0.04)$ & 4.19 & $(0.01)$ \\
\hline 5 & $\mathrm{~N}$ & 2.61 & $(0.20)$ & 3.36 & $(0.01)$ & 3.41 & $(0.00)$ & 3.66 & $(0.17)$ & 3.78 & $(0.01)$ \\
\hline 5 & $\mathrm{Q}$ & 2.94 & $(0.06)$ & 3.53 & $(0.02)$ & 3.57 & $(0.08)$ & 3.95 & $(0.01)$ & 4.13 & $(0.01)$ \\
\hline 6 & $\mathrm{~N}$ & 2.46 & $(0.24)$ & 3.08 & $(0.02)$ & 3.35 & $(0.03)$ & 3.53 & $(0.02)$ & 3.65 & $(0.01)$ \\
\hline 6 & Q & 2.67 & $(0.02)$ & 3.07 & $(0.00)$ & 3.66 & $(0.13)$ & 3.78 & $(0.02)$ & 3.87 & $(0.01)$ \\
\hline \multicolumn{12}{|c|}{ B3LYP } \\
\hline 1 & $\mathrm{~N}$ & 4.88 & $(0.00)$ & 5.57 & $(0.01)$ & 5.70 & $(0.02)$ & 6.28 & $(0.02)$ & 6.31 & $(0.00)$ \\
\hline 1 & $\mathrm{Q}$ & 5.56 & $(0.00)$ & 5.88 & $(0.00)$ & 6.07 & $(0.00)$ & 6.14 & $(0.00)$ & 6.63 & $(0.02)$ \\
\hline 2 & $\mathrm{~N}$ & 3.69 & $(0.22)$ & 4.32 & $(0.02)$ & 4.51 & $(0.05)$ & 4.63 & $(0.01)$ & 4.70 & $(0.04)$ \\
\hline 2 & Q & 4.84 & $(0.00)$ & 4.96 & $(0.00)$ & 5.03 & $(0.00)$ & 5.11 & $(0.00)$ & 5.22 & $(0.00)$ \\
\hline 3 & $\mathrm{~N}$ & 3.56 & $(0.23)$ & 4.16 & $(0.04)$ & 4.47 & $(0.01)$ & 4.58 & $(0.07)$ & 4.65 & $(0.01)$ \\
\hline 3 & $\mathrm{Q}$ & 4.55 & $(0.03)$ & 4.66 & $(0.04)$ & 4.80 & $(0.05)$ & 4.97 & $(0.02)$ & 5.07 & $(0.04)$ \\
\hline 4 & $\mathrm{~N}$ & 3.37 & & 4.10 & & 4.41 & & 4.48 & & 4.52 & $(0.08)$ \\
\hline 4 & Q & 4.25 & $(0.10)$ & 4.52 & $(0.01)$ & 4.70 & $(0.07)$ & 4.73 & $(0.02)$ & 4.96 & $(0.02)$ \\
\hline 5 & $\mathrm{~N}$ & 3.16 & $(0.27)$ & 4.06 & $(0.01)$ & 4.22 & $(0.03)$ & 4.33 & $(0.15)$ & 4.44 & $(0.01)$ \\
\hline 5 & Q & 3.91 & $(0.13)$ & 4.32 & $(0.12)$ & 4.47 & $(0.01)$ & 4.73 & $(0.02)$ & 4.87 & $(0.02)$ \\
\hline 6 & $\mathrm{~N}$ & 3.04 & $(0.28)$ & 3.82 & $(0.04)$ & 4.11 & $(0.05)$ & 4.21 & $(0.01)$ & 4.33 & $(0.05)$ \\
\hline 6 & Q & 3.62 & $(0.03)$ & 4.04 & $(0.01)$ & 4.30 & $(0.03)$ & 4.38 & $(0.14)$ & 4.53 & $(0.06)$ \\
\hline \multicolumn{12}{|c|}{ CAM-B3LYP } \\
\hline 1 & $\mathrm{~N}$ & 5.23 & $(0.00)$ & 6.11 & $(0.03)$ & 6.20 & $(0.02)$ & 6.65 & $(0.03)$ & 6.86 & $(0.00)$ \\
\hline 1 & Q & 6.06 & $(0.00)$ & 6.33 & $(0.00)$ & 6.60 & $(0.00)$ & 6.67 & $(0.00)$ & 7.24 & $(0.02)$ \\
\hline 2 & $\mathrm{~N}$ & 4.17 & $(0.24)$ & 4.90 & $(0.02)$ & 5.15 & $(0.00)$ & 5.28 & $(0.09)$ & 5.43 & $(0.11)$ \\
\hline 2 & Q & 5.39 & $(0.00)$ & 5.41 & $(0.00)$ & 5.78 & $(0.02)$ & 5.83 & $(0.09)$ & 6.00 & $(0.02)$ \\
\hline 3 & $\mathrm{~N}$ & 4.08 & $(0.26)$ & 4.73 & $(0.05)$ & 5.03 & $(0.01)$ & 5.31 & $(0.12)$ & 5.39 & $(0.17)$ \\
\hline 3 & $\mathrm{Q}$ & 5.02 & $(0.02)$ & 5.23 & $(0.00)$ & 5.38 & $(0.17)$ & 5.66 & $(0.23)$ & 5.82 & $(0.01)$ \\
\hline 4 & $\mathrm{~N}$ & 3.95 & $(0.27)$ & 4.73 & $(0.04)$ & 5.00 & $(0.01)$ & 5.24 & $(0.16)$ & 5.38 & $(0.18)$ \\
\hline 4 & $\mathrm{Q}$ & 5.01 & $(0.03)$ & 5.14 & $(0.22)$ & 5.17 & $(0.00)$ & 5.60 & $(0.24)$ & 5.83 & $(0.02)$ \\
\hline 5 & $\mathrm{~N}$ & 3.79 & $(0.33)$ & 4.69 & $(0.03)$ & 4.95 & $(0.03)$ & 5.08 & $(0.27)$ & 5.27 & $(0.00)$ \\
\hline 5 & $\mathrm{Q}$ & 4.80 & $(0.36)$ & 5.03 & $(0.03)$ & 5.06 & $(0.03)$ & 5.49 & $(0.12)$ & 5.62 & $(0.14)$ \\
\hline 6 & $\mathrm{~N}$ & 3.72 & $(0.36)$ & 4.44 & $(0.05)$ & 4.84 & $(0.02)$ & 4.93 & $(0.08)$ & 5.05 & $(0.14)$ \\
\hline 6 & Q & 4.57 & $(0.03)$ & 4.84 & $(0.20)$ & 4.95 & $(0.07)$ & 5.18 & $(0.02)$ & 5.26 & $(0.27)$ \\
\hline
\end{tabular}



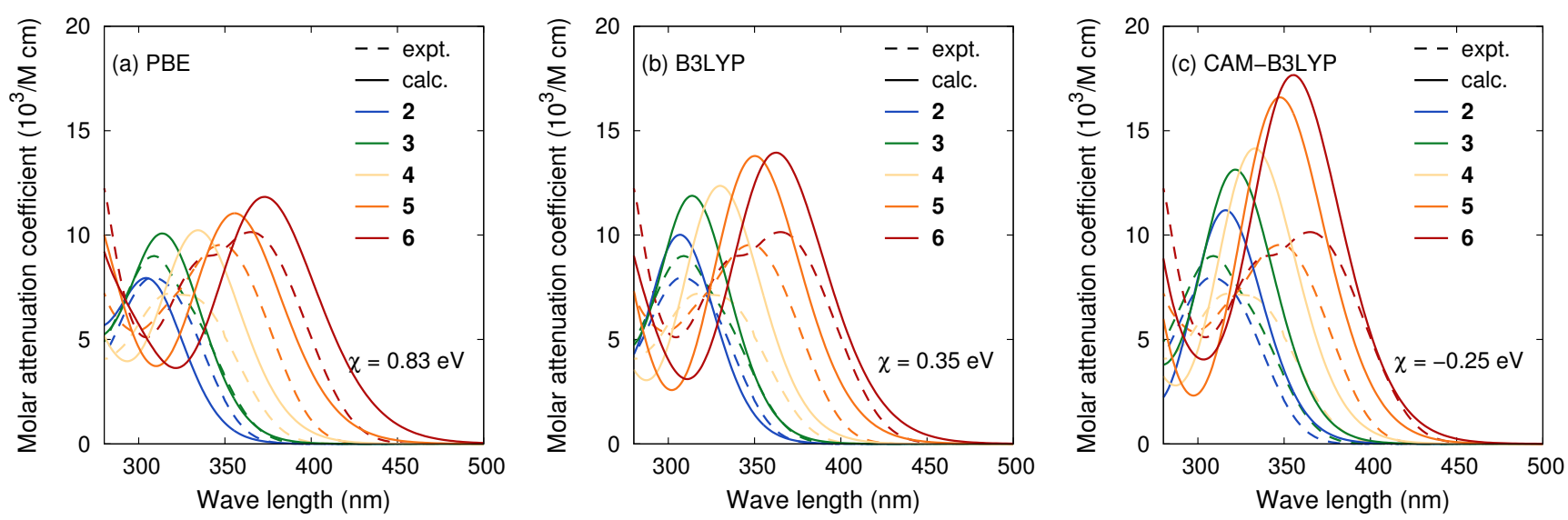

Figure 1: Comparison of the molar attenuation coefficients of $\mathbf{2}-\mathbf{6}$ from experiment ${ }^{4}$ and (a) PBE, (b) B3LYP, and (c) CAM-B3LYP calculations. A smearing using a width of $0.25 \mathrm{eV}$ was applied to the latter to mimic the broadening due to vibrations. The calculated spectra are unshifted.

with the exception of CASPT2 and $\operatorname{CCSD}(\mathrm{T})$, for which single-point calculations were carried out based on CASSCF and MP2 geometries, respectively. All calculations are performed with NWChem $6.5^{5}$ except for CASSCF and CASPT2, which were performed with MolCas 8.0. ${ }^{6-8}$ The MP2 results were extrapolated to the complete basis set (CBS) limit as suggested by Halkier et al. ${ }^{9,10}$ while the $\operatorname{CCSD}(\mathrm{T})$ data were extrapolated to the CBS limit following Jurecka et al. ${ }^{11}$. The results of our calculations for $\Delta E$ are compiled in Table 7 .

The experimental reference value for $\Delta H$ is $92 \pm 1 \mathrm{~kJ} / \mathrm{mol}$. To obtain $\Delta E$ we substract the $\Delta H-\Delta E$ difference obtained at the DFT level, i.e.

$$
\Delta E_{\text {expt }}=\Delta H_{\text {expt }}-\left(\Delta H_{\mathrm{DFT}}-\Delta E_{\mathrm{DFT}}\right) .
$$

Using B3LYP data (see main text), we then obtain $\Delta E_{\text {expt }}=94 \pm 1 \mathrm{~kJ} / \mathrm{mol} .{ }^{12}$ The extrapolated $\operatorname{CCSD}(\mathrm{T})$ value of $\Delta E=95.5 \mathrm{~kJ} / \mathrm{mol}$, which represents the highest level of calculation among our calculated data, is in fact in very good agreement with the experimental value. Both $\Delta E_{\text {expt }}$ and $\Delta E_{\mathrm{CCSD}(\mathrm{T})}$ are shown as reference values in Fig. 4(b) of the main article.

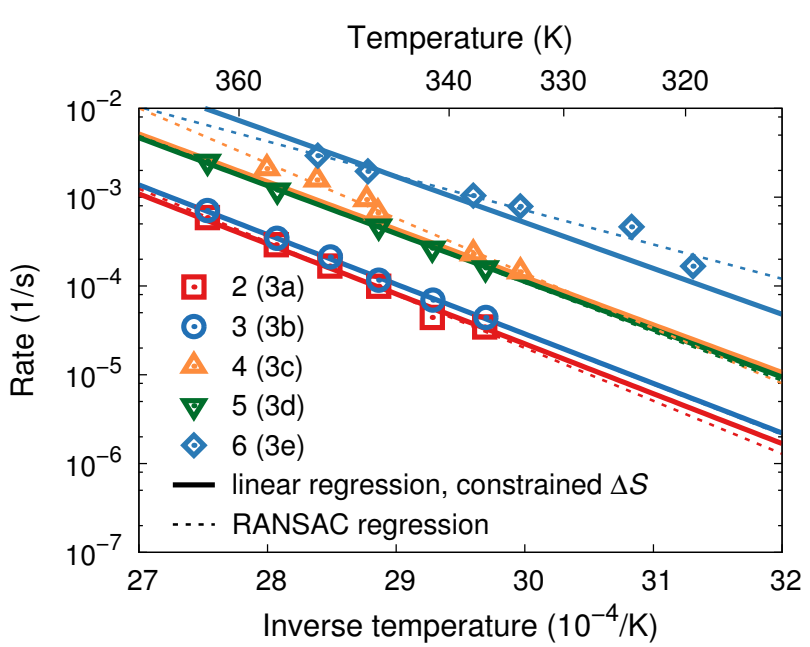

Figure 2: Comparison of different approaches for fitting the experimental data for thermal $\mathrm{Q} \rightarrow$ back-conversion. While the assessment indicates a certain level of uncertainty regarding the thus obtained barriers, it highlights the systematic variation of the conversion rates across the compounds, which are ordered by onset of absorption. For conveniences, the numbers in the legend given in brackets refer to the compound enumeration used in Gray et al. ${ }^{4}$. 
Table 7: Storage energies (electronic contribution only) for the unsubstituted norbornadiene-quadricylane system at different levels of calculation.

\begin{tabular}{|c|c|c|}
\hline Method & Basis set & $\Delta E(\mathrm{~kJ} / \mathrm{mol})$ \\
\hline M06 & $6-311+\mathrm{G}^{*}$ & 54.73 \\
\hline PBE & $6-311+\mathrm{G}^{*}$ & 71.06 \\
\hline B3LYP & $6-311+\mathrm{G}^{*}$ & 99.71 \\
\hline B3LYP & aug-cc-pvdz & 91.62 \\
\hline B3LYP & aug-cc-pvtz & 96.80 \\
\hline MP2 & aug-cc-pvdz & 93.76 \\
\hline MP2 & aug-cc-pvtz & 86.03 \\
\hline MP2 & aug-cc-pvqz & 83.89 \\
\hline MP2 & $\left(\right.$ extrapolated $\left.^{a}\right)$ & 82.59 \\
\hline CASPT2//CASSCF & ANO-S DZP & 97.91 \\
\hline CASPT2//CASSCF & aug-cc-pvdz & 95.22 \\
\hline $\operatorname{CCSD}(\mathrm{T}) / / \mathrm{MP} 2$ & aug-cc-pvdz & 106.02 \\
\hline $\operatorname{CCSD}(\mathrm{T}) / / \mathrm{MP} 2$ & aug-cc-pvtz & 98.92 \\
\hline $\operatorname{CCSD}(\mathrm{T})$ & $\left(\right.$ extrapolated $\left.^{b}\right)$ & 95.48 \\
\hline
\end{tabular}

${ }^{a}$ Extrapolation of MP2 data to basis set limit ${ }^{9,10}$

${ }^{b}$ Extrapolation of $\operatorname{CCSD}(\mathrm{T})$ data to MP2 basis set limit. ${ }^{11}$

\section{Experimental back-conversion barriers}

The thermal stability of the substituted compounds considered in the present study has been previously investigated experimentally. ${ }^{4}$ To this end, the absorbance increase associated with the $\mathrm{N}$ variant was measured as a function of time at different temperatures. The resulting data was fitted to the Eyring equation to extract the enthalpy barriers for backconversion along with the associated entropies. The thus obtained data exhibits notable error bars and a pronounced correlation between entropy and enthalpy. Furthermore, the halflife times, which compound enthalpies and entropies, exhibit a very noticeable correlation with the onset of absorption; this is very much unlike the barriers for which no clear trend can be observed. These observations alongside the arguments put forth in the "Transition state" section of the paper prompted us to reinspect these data.

In this context, we refitted the experimental data for the time dependence of the absorbance using both the random sample con- sensus (RANSAC) method as implemented in scikit-learn ${ }^{13}$ and constrained linear regression. The RANSAC algorithm is designed to account for data with outliers, albeit typically more suitable for larger data sets. In the case of the constrained linear regression, we fitted the data sets for all five compounds simultaneously using different enthalpies for each compound but only one value for the entropy. Both approaches yield good representations of the data of equal or better quality than the original fit (Fig. 2 and Table II of the main paper). While this illustrates the difficulty of obtaining reliable values for the barriers, more importantly it demonstrates that the conversion enthalpies and the onset of absorption (or alternatively the absorption maximum) are indeed correlated with each other (Fig. 7 of the main paper).

\section{References}

(1) Knuchel, G.; Grassi, G.; Vogelsanger, B.; Bauder, A. Molecular Structure of Norbornadiene as Determined by Microwave Fourier Transform Spectroscopy. J. Am. Chem. Soc. 1993, 115, 10845. 
(2) Akimichi, Y.; Kozo, K. Structures of Norbornane and Norbornadiene as Determined by Gas Electron Diffraction. Bull. Chem. Soc. Jpn. 1971, 44, 2356.

(3) Mizuno, K.; Fukuyama, T.; Kuchitsu, K. Molecular Structure of Quadricyclane (Tetracyclo[3.2.0.0 $\left.0^{2,7} \cdot 0^{4,6}\right]$ Heptane) Studied by Gas Electron Diffraction. Chem. Lett. 1972, 1, 249.

(4) Gray, V.; Lennartson, A.; Ratanalert, P.; Börjesson, K.; Moth-Poulsen, K. Diarylsubstituted Norbornadienes With Redshifted Absorption for Molecular Solar Thermal Energy Storage. Chem. Commun. 2014, 50, 5330-5332.

(5) Valiev, M.; Bylaska, E. J.; Govind, N.; Kowalski, K.; Straatsma, T. P.; Van Dam, H. J. J.; Wang, D.; Nieplocha, J.; Apra, E.; Windus, T. L. et al. NWChem: A Comprehensive and Scalable Open-source Solution for Large Scale Molecular Simulations. Comp. Phys. Comm. 2010, 181, 1477-1489.

(6) Aquilante, F.; De Vico, L.; Ferré, N.; Ghigo, G.; Malmqvist, P.-A.; Neogrády, P.; Pedersen, T. B.; Pitoňák, M.; Reiher, M.; Roos, B. O. et al. MOLCAS 7: The Next Generation. J. Comp. Chem. 2010, 31, 224-247.

(7) Veryazov, V.; Widmark, P.-O.; SerranoAndrés, L.; Lindh, R.; Roos, B. O. MOLCAS as a Development Platform for Quantum Chemistry Software. Int. J. Quantum Chem. 2004, 100, 626-635.

(8) Karlström, G.; Lindh, R.; Malmqvist, P.A.; Roos, B. O.; Ryde, U.; Veryazov, V.; Widmark, P.-O.; Cossi, M.; Schimmelpfennig, B.; Neogrady, P. et al. MOLCAS: A Program Package for Computational Chemistry. Comp. Mater. Sci. 2003, 28, 222-239.

(9) Halkier, A.; Helgaker, T.; Jørgensen, P.; Klopper, W.; Koch, H.; Olsen, J.; Wilson, A. K. Basis-set Convergence in Cor- related Calculations on $\mathrm{Ne}, \mathrm{N}_{2}$, and $\mathrm{H}_{2} \mathrm{O}$. Chem. Phys. Lett. 1998, 286, 243-252.

(10) Halkier, A.; Helgaker, T.; Jørgensen, P.; Klopper, W.; Olsen, J. Basis-set Convergence of the Energy in Molecular Hartree-Fock Calculations. Chem. Phys. Lett. 1999, 302, 437-446.

(11) Jurecka, P.; Sponer, J.; Cerny, J.; Hobza, P. Benchmark Database of Accurate (MP2 and CCSD(T) Complete Basis Set Limit) Interaction Energies of Small Model Complexes, DNA Base Pairs, and Amino Acid Pairs. Phys. Chem. Chem. Phys. 2006, 8, 1985-1993.

(12) An, X.; Xie, Y. Enthalpy of Isomerization of Quadricyclane to Norbornadiene. Thermochimica Acta 1993, 220, 17.

(13) scikit-learn. http://scikit-learn.org/; accessed 2016/01/18. 